\title{
Standardization of FISH-procedures: Summary of the first discussion workshop
}

\author{
Michael Hausmann ${ }^{\mathrm{a}, \mathrm{b}, *}$ and Christoph Cremer ${ }^{\mathrm{a}}$ \\ ${ }^{a}$ Kirchhoff Institute of Physics, University of Heidelberg, Im Neuenheimer Feld 227, D-69120 Heidelberg, \\ Germany \\ ${ }^{\mathrm{b}}$ Institute of Pathology, University Hospital, University of Freiburg, Albertstr. 19, D-79104 Freiburg, Germany
}

Received 10 June 2003

Accepted 8 July 2003

\section{Introduction}

Currently, fluorescent in situ hybridization (FISH) is widely used to assess the localization of genetic elements in tissues, nuclei of cultured cells, and spreads of metaphase chromosomes. The advent of commercially available probes and labelling kits for whole or partial chromosome painting and pathogenetically important gene loci has allowed FISH to enter routine work. Still, however, FISH procedures vary grossly from laboratory to laboratory, and are far from optimal for many questions in modern biology and medicine.

Here, a report on the 1st discussion workshop on standardization of FISH-procedures held at Schloss Elmau, Bavaria, October 9-10, 2002 is given. This meeting was organised by Christoph Cremer (Heidelberg), Michael Hausmann (Freiburg) and Hans J. Tanke (Leiden) as a satellite workshop to the 2nd Elmau conference on nuclear organization. The workshop was convented to discuss recent developments, problems of routine applications, and future requirements in the intriguing subject of specific fluorescence DNA labelling especially in the interphase cell nucleus. The idea was to bring together applicants of diagnostic routine, applicants in basic cytogenetic research, and developers of FISH techniques. The 10 participants very lively supported the discussion and elaborated some future aspects for methodological research and requirements to FISH probe manufacturers.

\footnotetext{
*Corresponding author: PD Dr. Michael Hausmann, Pathologisches Institut, Postfach, D-79002 Freiburg, Germany. Tel.: +49 761 2036780; Fax: +49 761 2036790; E-mail: mihaus@ukl.unifreiburg.de.
}

\section{Methodological developments}

After opening remarks, Christoph Cremer gave an overview over the principle of FISH. At a first glimpse, such an introduction to a nowadays widely used technique appears to be superfluous. Nonetheless, in reality the process to attach fluorescence labelled nucleic acid sequences to their complementary counterparts in the genome is much more complex than textbook figures tend to suggest. A better understanding of the physics of FISH is therefore expected to contribute to the further development of this technique for practical applications. Such improvements appear to be highly desirable. For example, under certain circumstances in routine use it may be a serious problem that FISH procedures take about two days inclusive overnight hybridization of probe and target. The application of chaotropic agents like formamide causes a series of washing steps. This procedure may therefore considerably influence the chromosomal morphology [14]. On the basis of systematic investigations of the denaturation step of the chromosomal target in the FISH protocol [12,15], a new FISH technique was developed omitting several complex steps in the protocol. In this novel Fast-FISH protocol [3] chaotropic agents for denaturation were omitted. Denaturation and probe to target hybridization were only controlled by temperature and duration of heat treatment, so that the whole procedure was substantially accelerated $[6,9]$.

Fast-FISH was applied for specific labelling with centromere probes [3] and for chromosome painting with repeat depleted probes [5]. The usefulness of FastFISH was proven in hemato-oncology for the detec- 
tion of numerical aberrations in interphase cell nuclei of CLL and AML patients [8] and for the detection of dicentric metaphase-chromosomes after ionising radiation exposure in biological dosimetry [6]. Nevertheless, in these Fast-FISH protocols using commercially available centromere probes, labelling of minor binding sites could occur. In such cases it was shown that this shortcoming was overcome by means of digital microscopy and appropriate procedures for image analysis.

In a first conclusion, Christoph Cremer pointed out the necessity of appropriate digital image analysis in combination with the Fast-FISH procedure used. It seems to be highly important to optimize not only the FISH protocols but to adapt procedures of automated microscopy and digital image analysis in order to increase the contrast and FISH efficiency.

In a second plenary talk Michael Hausmann addressed the influence of thermal denaturation and FISH on the chromosomal morphology [14]. He presented temperature dependent hyperchromicity measurements at $256 \mathrm{~nm}$ and $313 \mathrm{~nm}$ which were performed to analyze the thermal denaturation behaviour with and without formamide of metaphase chromosome suspensions [12]. Absorbance changes were measured between $20^{\circ} \mathrm{C}$ and $95^{\circ} \mathrm{C}$ with a temperature gradient of $1^{\circ} \mathrm{C} / \mathrm{min}$ using an appropriately designed computer-controlled photometer device. The denaturation profiles or melting profiles (= first derivative of the absorbance curve) resulted in a highly variable peak pattern indicating complex conformation changes. The influence of formamide on the denaturation behaviour depended on the duration of the formamide exposure. To further analyse the influence of thermal denaturation on the chromosomal morphology, a non-enzymatic, low temperature FISH (LTFISH) procedure was developed and applied to metaphase spreads $[4,14]$. Low temperature means in this context that the usually applied denaturation procedure of the chromosomal target DNA by heat treatment and formamide was completely omitted so that the complete hybridization reaction took place at $37^{\circ} \mathrm{C}$. For the DNA probe pUC 1.77 specific for the chromosome region 1q12, LTFISH was performed. After 15 hours hybridization time, the chromosomal morphology was analysed by scanning near-field optical microscopy (SNOM) [10] and compared with the chromosomal morphology after (a) labelling of all centromeres using the same chemical treatment in the FISH procedure but with the application of target denaturation and after (b) labelling using a standard FISH protocol inclu- sive thermal denaturation of the DNA probe and the chromosomal target. Depending on the applied FISHprocedure, SNOM images showed substantial differences of the chromosome morphology. After LTFISH the chromosome morphology appeared to be much better preserved than after standard FISH. The application of the LTFISH chemical treatment accompanied by heat denaturation had a very destructive influence on the chromosomal morphology [14].

LTFISH unfortunately does not work for any given FISH probe. Therefore, further developments towards FISH procedures without target denaturation were performed. Using a specific combination of homopurine or homopyrimidine oligo probes, genome loci may be labelled by means of triple strand binding of the probe [2]. Although these developments are in the very beginning and only preliminary results were presented, this new technique called COMBO-FISH (COMBinatorical Oligo FISH) appeared to be a promising methodological advance towards the long term but still elusive goal of carrying out FISH in high-resolution microscopy of vital cells [11]. COMBO-FISH might be useful not only for better specimen preservation but also for improved specificity in Fast-FISH applications including heat denaturation: the human DNA sequence data base allows the computer selection of a number of non-repetitive oligo sequences for any given small genome region down to the level below $100 \mathrm{~kb}$. Such regions are small enough that all fluorescence photons emitted from the various labelled oligo nucleotides bound to the complementary genome sites would merge into one diffraction limited light microscopic signal $[2,11]$.

\section{Open questions}

After these introductionary talks, several subjects of discussion were defined:

- the usefulness of the so called standard protocols with probe and target denaturation;

- alternative approaches with "in vivo" labels maintaining cell vitality (e.g., GFP-labelling, sequence specific proteins, COMBO-FISH, etc.);

- applied microscope systems (sensitivity, resolution, etc.);

- computer image analysis (automation vs. manual operation);

- requirements for standardization or optimization. 
In this context the central question was discussed, how much optical resolution is required or what is the useful scale for specimen analysis. The researcher needs a preservation of the nano-structure to such an extent necessary to the biomedical question to be answered. For example, if the cell-by-cell transcription potential of a given gene is to be monitored by the analysis of its compaction, even a relatively modest conservation of the nanostructure might be sufficient. In any case a sensitive labelling and the best microscopic resolution are desirable. On the other hand, the requirements for clinicians can be summarized with the words "robust" and "fast" which includes the demand of high reproducibility and easy handling.

\section{Problems of clinical routine}

A typical problem in clinical routine is the conflict between protocol optimization and protocol standardization which was described by examples from the institute of pathology, Kassel. A new commercially available probe kit for the gene Her2/neu was applied in routine pathology. Since it was an FDA approved probe kit, the protocol was standardized in all experimental details and could not be modified in order to optimize the protocol for certain types of specimen. However, the critical step that is not standardized is the pre-treatment and fixation. All pathological institutes have their own, for many years established procedures for fixation (e.g., formalin, buffered formalin, alcohol, Bouin's solution, etc.) which are variable factors, which are of different quality, and which are variable concerning the subsequent FISH procedure. Care must be taken that the fixative is in good condition and not degraded as it can easily be the case with formaldehyde fixatives; naturally, the tissue slice should be of a size that allows good penetration of the fixative; concentration of cross-linking fixatives and times of incubation should not be too long, otherwise penetration of probes will be hampered. In direct dependency of fixation are other conditions of pre-treatment, in particular protein digestion. The type of enzyme and incubation time are critical. Further hydrolysis with $\mathrm{HCl}$ may be necessary. If the protocol would not be standardized, several parameter would be open for optimization with reference to the fixation and pre-treatment protocol. However, the FDA-approval stands against such an optimization.

The discussion showed that clinical routine has to consider especially legal problems which exist with the consequences of a diagnosis. Therefore, existing standardized protocols cannot be changed, although the results may be of poor quality as compared to an optimized protocol. In order to overcome such problems, the so far subjective specimen inspection should also be optimized by computer image analysis. This would have the advantage that the subjective judgement "good quality" - "poor quality" would become measurable by the quantification of image parameters.

For instance, images can be calibrated according to intensity and size of fluorescence sites, chromatic effects due to specimen thickness, instrumentation parameter, etc. For this purpose C. Cremer suggested the application of appropriate standards manufactured by nano-lithography. This would allow the comparability of results obtained in different laboratories with different instrumentation equipment and variable specimen pre-treatment.

\section{Future requirements for research}

(a) Instrumentation: So far the commercially available microscopes are general purpose instruments in routine applications. High resolution systems have been only built in a very few laboratories that are extremely specialized for research purposes as, for instance, to measure the degree of chromatin condensation at small sites in the interphase cell nucleus [1]. The discussion showed that in the future also commercial instruments may reach a level towards a more special purpose design. Typical features for optimization are resolution, speed, automatization, specimen accessibility, sensitivity, robustness, software handling, etc. These features have to be optimized according to the individual needs of the user and not in general for all instruments. For instance, for clinicians the factor robustness may be of prominent importance whereas resolution may be of lower importance in many applications. On the other hand the cytogeneticist who is working in basic research, often needs the best resolution that can be obtained for his specimens and does not require the same robustness for the instrument as his collegue in clinical routine.

(b) FISH protocols: A general standardization of FISH protocols appears to be impossible. For researchers new developments towards "vital" FISH labelling are of high interest. Nevertheless, in vivo approaches are not necessary in general. Such developments are presently not of major importance for diagnostics, although there is still some need for gentle 
FISH procedures. Concerning the cost of FISH in routine, it is important to develop "easy" techniques with simplified steps in the protocol, to increase the sensitivity for microscopic inspection, and to accelerate the procedure. Protocols for clinical routine should also consider legal problems. Here, the leading companies for FISH probes have the task to make the clinical studies and to obtain appropriate permissions, for instance, FDA-approval.

\section{Conclusion}

Apart from further increasing the robustness and ease of use to enhance the applicability of FISH, future developments of FISH procedures should consider the importance of three-dimensional structure, especially under the aspect of the functional correlation of nuclear architecture and genetic activity. Clearly, a compromise must be found between the preservation of cellular nanostructure, and the accessibility for probes. To this end, it will be vital to define the resolution that is sensible in assessing nuclear structures. Development of innovative light microscopy methods has improved the possible resolution down to a few tens of nanometers $[1,7,13]$, but it is questionable if such high resolutions can provide more information in biological specimens when using the presently available standard FISH techniques.

This first discussion workshop on standardization of FISH has shown that although FISH has become a routine technique there is need for further methodological developments. This includes

(a) novel techniques for basic research (e.g., FISH in vital cells);

(b) improved standardization for clinical routine (such a standardization might be possible not only on the level of the FISH procedure itself but also on the level of the resulting images, where specific problem-related image quality might be standardized);

(c) developments in instrumentation and image analysis software with respect to special conditions of FISH protocols (acceptance of FISH results depending on image quality).

To continue the discussion, a second workshop will be held in July 2003; it should bring together scientists, clinicians and members of companies for FISH probe production and companies for microscopic instrumentation.

\section{Acknowledgements}

The authors gratefully acknowledge the contribution of the participators of the discussion workshop. The authors thank Christian Schöfer, Vienna, and Frank O. Fackelmayer, Hamburg, for their contributions to the final summarizing text. Furthermore, the organizers of the workshop want to express their tanks to Margund Bach, Heidelberg, who did all the secretary work and other organisation details for the success of the workshop.

\section{References}

[1] B. Albrecht, A.V. Failla, A. Schweitzer and C. Cremer, Spatially modulated illumination microscopy: A new approach to biological nanostructure analysis. G.I.T., Imaging \& $\mathrm{Mi}$ croscopy 2 (2001), 40-42.

[2] C. Cremer, M. Hausmann and T. Cremer, Markierung von Nukleinsäuren mit speziellen Probengemischen (Labelling of nucleic acids by specific probe mixtures) (1997) Offenlegungsschrift DE 19806962 A1, Deutsches Patentamt, München (Offenlegungstag 1.10.1998).

[3] M. Durm, F.-M. Haar, M. Hausmann, H. Ludwig and C. Cremer, Optimization of fast-fluorescence in situ hybridization with repetitive $\alpha$-satellite probes, Z. Naturforsch. C 51 (1996), 253-261.

[4] M. Durm, F.-M. Haar, M. Hausmann, H. Ludwig and C. Cremer, Non-enzymatic, low temperature fluorescence in situ hybridization of human chromosomes with a repetitive $\alpha$-satellite probe, Z. Naturforsch. C 52 (1997), 82-88.

[5] M. Durm, L. Schüssler, H. Münch, J. Craig, H. Ludwig, M. Hausmann and C. Cremer, Fast-painting of human metaphase spreads using a chromosome specific repeat depleted DNA library probe, Biotechniques 24 (1998), 820-825.

[6] M. Durm, I. Sorokine-Durm, F.-M. Haar, M. Hausmann, H. Ludwig, P. Voisin and C. Cremer, Fast-FISH technique for rapid, simultaneous labeling of all human centromeres, Cytometry 31 (1998), 153-162.

[7] A. Egner, S. Jakobs and S.W. Hell, Fast 100-nm resolution three-dimensional microscope reveals structural plasticity of mitochondria in live yeast, Proc. Natl. Acad. Sci. USA 99 (2002), 3370-3375.

[8] A. Esa, L. Trakhtenbrot, M. Hausmann, J. Rauch, F. BrokSimoni, G. Rechavi, I. Ben-Bassat and C. Cremer, Fast-FISH detection and automated image analysis of numerical chromosome aberrations in hematological malignancies, Analyt. Cell. Pathol. 16 (1998), 211-222.

[9] F.-M. Haar, M. Durm, M. Hausmann, H. Ludwig and C. Cremer, Optimization of Fast-FISH for $\alpha$-satellite DNA probes, J. Biochem. Biophys. Methods 33 (1996), 43-54.

[10] M. Hausmann, B. Perner, A. Rapp, H. Scherthan and K.O. Greulich, SNOM imaging of mitotic and meiotic chromosomes, Eur. Microsc. Analys. 71(5) (2001), 5-7. 
[11] M. Hausmann, R. Winkler, G. Hildenbrand, J. Finsterle, A. Weisel, A. Rapp, E. Schmitt, S. Janz and C. Cremer, COMBOFISH: specific labelling of non-denatured chromatin targets by computer selected DNA oligo-probe combinations, Biotechniques (in press).

[12] J. Rauch, D. Wolf, M. Hausmann and C. Cremer, The influence of formamide on thermal denaturation profiles of DNA and metaphase chromosomes in suspensions, Z. Naturforsch. $C$ 55 (2000), 737-746.

[13] J. Szollosi, P. Nagy, Z. Sebestyen, S. Damjanovicha, J.W. Park and L. Matyus, Applications of fluorescence resonance energy transfer for mapping biological membranes, J. Biotechnol. 82 (2002), 251-266.
[14] R. Winkler, B. Perner, A. Rapp, M. Durm, C. Cremer, K.O. Greulich and M. Hausmann, Labelling quality and chromosome morphology after low temperature FISH analysed by scanning far-field and scanning near-field optical microscopy, J. Microsc. 209 (2003), 23-33.

[15] D. Wolf, J. Rauch, M. Hausmann and C. Cremer, Comparison of the thermal denaturation behaviour of DNA-solutions and chromosome preparations in suspension, Biophys. Chem. 81 (1999), 207-221. 


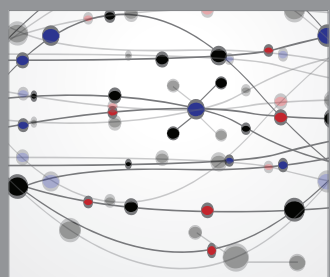

The Scientific World Journal
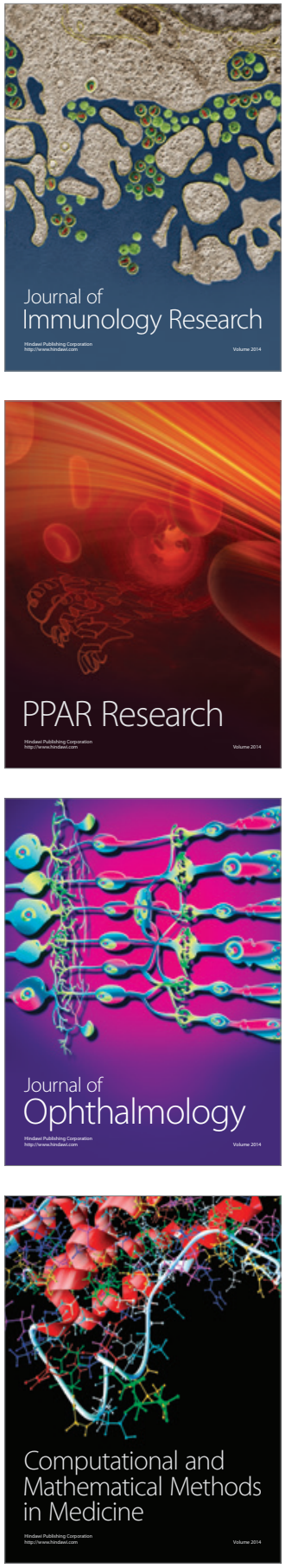

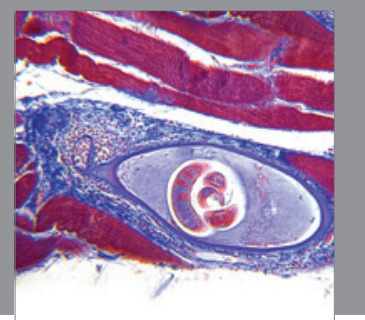

Gastroenterology

Research and Practice
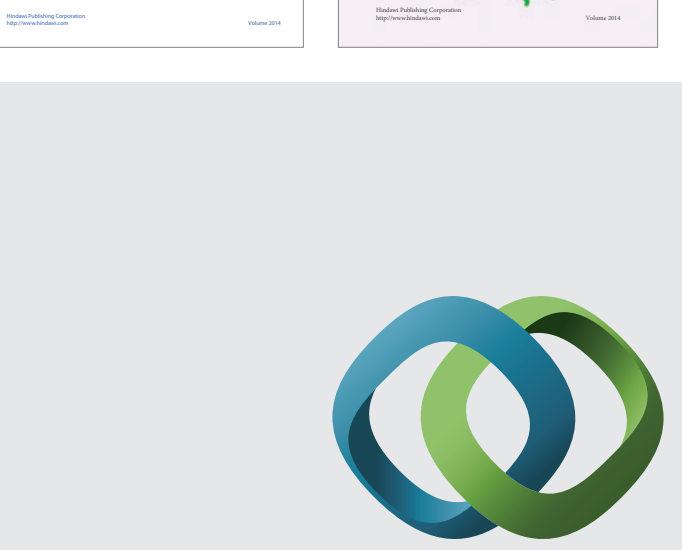

\section{Hindawi}

Submit your manuscripts at

http://www.hindawi.com
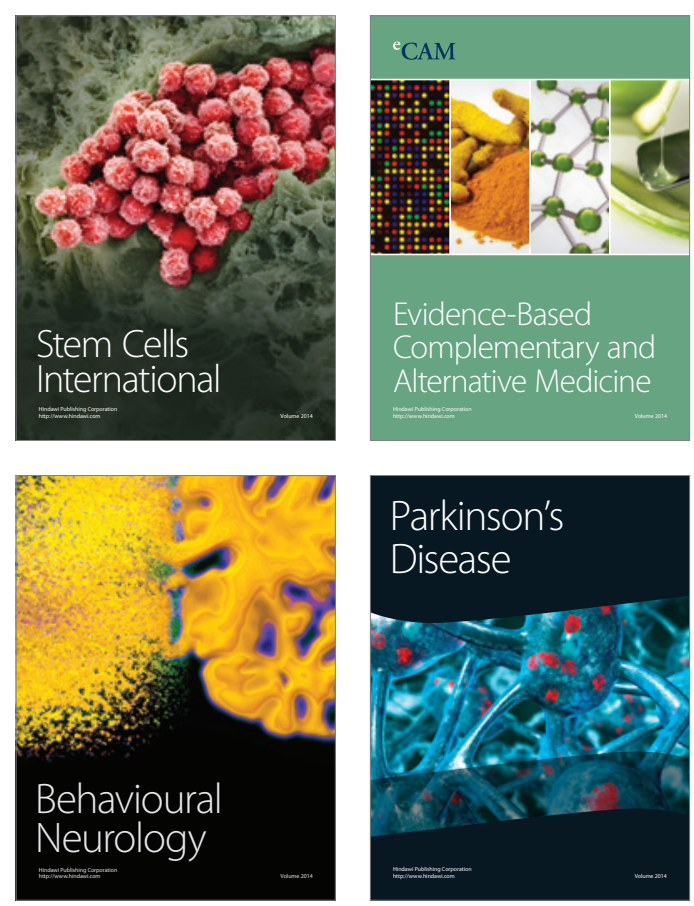

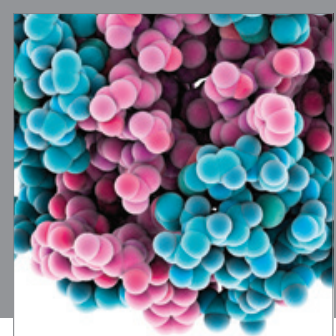

Journal of
Diabetes Research

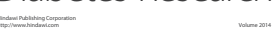

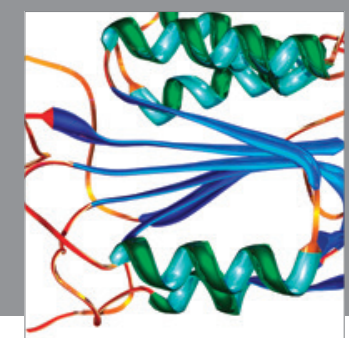

Disease Markers
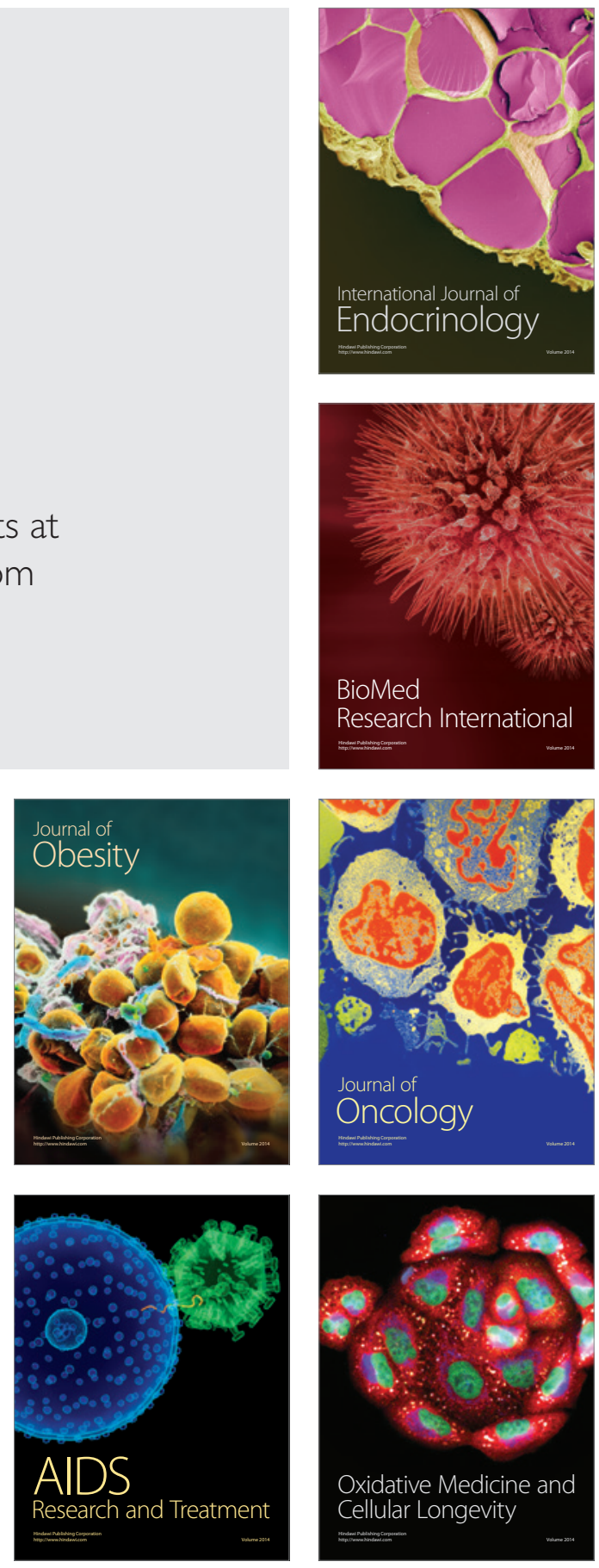